\title{
Insights into 3D Structure of ADAMTS13: A Stepping Stone towards Novel Therapeutic Treatment of Thrombotic Thrombocytopenic Purpura
}

\author{
Bogac Ercig ${ }^{1,2,3, *}$ Kanin Wichapong ${ }^{1}$ Chris P. M. Reutelingsperger ${ }^{1,3, *}$ Karen Vanhoorelbeke K,* $^{4, *}$ \\ Jan Voorberg ${ }^{2, *}$ Gerry A.F. Nicolaes ${ }^{1,3, *}$
}

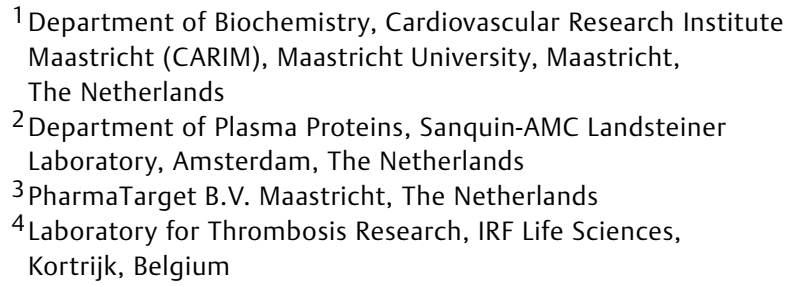

Address for correspondence Gerry A.F. Nicolaes, PhD, Department of Biochemistry, Cardiovascular Research Institute Maastricht, CARIM, Maastricht University, 6200MD Maastricht, The Netherlands (e-mail: g.nicolaes@maastrichtuniversity.nl).

Thromb Haemost 2018;118:28-41.

\begin{abstract}
Keywords

- ADAMTS13

- von Willebrand factor

- thrombosis

- thrombotic thrombocytopenic purpura

- autoimmune diseases

ADAMTS13 (a disintegrin and metalloprotease with a thrombospondin type-1 motif, member 13) and von Willebrand factor (VWF) can be considered as scale weights which control platelet adhesion during primary haemostasis. In a very uncommon condition designated thrombotic thrombocytopenic purpura (TTP), functional absence of ADAMTS13 tips the balance toward VWF-mediated platelet adhesion in the microcirculation. TTP is associated with a high mortality and arises from either a congenital or acquired autoimmune deficiency of the plasma enzyme ADAMTS13. In case of acquired ADAMTS13 deficiency, autoantibodies bind to and inhibit the function of ADAMTS13. Currently available treatments of TTP aim to supply ADAMTS13 through plasma exchange or are aimed at B-cell depletion with rituximab. None of the available therapeutics, however, aims at protection of ADAMTS13 from circulating autoantibodies. In this review, our aim is to describe the structure-function relationship of ADAMTS13 employing homology models and previously published crystal structures. Structural bioinformatics investigation of ADAMTS13 reveals many insights and explains how mutations and autoantibodies may lead to the pathophysiology of TTP. The results of these studies provide a roadmap for the further development of rationally designed therapeutics for the treatment of patients with acquired TTP. In addition, we share our opinion on the state of the art of the open-closed conformations of ADAMTS13 which regulate the activity of this highly specific VWF cleaving protease.
\end{abstract}

On behalf of the PROFILE Consortium, http://www.itn-profile.eu/

received

June 12, 2017

accepted after revision

October 13, 2017
Copyright @ 2018 Schattauer
DOI https://doi.org/

10.1160/TH17-06-0404.

ISSN 0340-6245. 


\section{Introduction}

Thrombotic thrombocytopenic purpura (TTP) is a rare thrombotic microangiopathy (TMA) with an estimated incidence of 3.7 cases per million per year. ${ }^{1}$ TTP is the result of the formation of thrombi in the microcirculation that hampers microcirculatory blood flow resulting in organ damage. The underlying mechanism of thrombus formation involves the impairment of proteolytic processing of secreted von Willebrand factor (VWF) giving rise to ultra-large von Willebrand factor multimers (ULVWF) in the microcirculation. ${ }^{2}$ Spontaneous binding of platelets to ULVWF eventually results in formation of thrombi that obstruct the microcirculation. Generated thrombi produce locally high shear stresses that can damage red blood cells resulting in anaemia and schistocyte formation, which is visible in blood smears from TTP patients. ${ }^{3}$ The formation of platelet-rich thrombi results in thrombocytopenia which presents as small skin bleeds known as petechiae: one of the diagnostic hallmarks of TTP. ${ }^{4}$ Besides thrombocytopenia and haemolytic anaemia, TTP can present with a variety of other clinical features which include liver and kidney damage as well as neurological abnormalities. ${ }^{3,5}$

The protease ADAMTS13 (a disintegrin and metalloprotease with a thrombospondin type- 1 motif, member 13) processes secreted VWF and prevents accumulation of ULVWF in the microcirculation. ADAMTS13 activity is low in TTP patients and its deficiency can be hereditary or can arise from the development of autoantibodies. Hereditary forms of ADAMTS13 deficiency (also described as UpshawSchulman syndrome) are seen in only $5 \%$ of all TTP patients. ${ }^{6}$ In approximately $95 \%$ of the patients, an acquired immunemediated form of the disease is present, which results from the as yet unexplained development of antibodies that target ADAMTS13 in previously healthy individuals. Diseases such as cancer, ${ }^{7}$ hepatitis, ${ }^{8}$ infections ${ }^{9,10}$ or other autoimmune diseases, like systemic lupus erythematosus (SLE), ${ }^{11}$ can also be followed by secondary TTP.

TTP is a fatal disease that, if untreated, causes mortality rates of up to $90 \%$. Diagnoses are generally made through careful analysis of routine clinical laboratory data, supplemented by specialized laboratory testing. To date, targeted specific therapeutics are not available and the main treatment for acquired TTP is plasma exchange therapy (PEX) which results in a drop of mortality rates to 10 to $20 \% .^{12}$ PEX promotes temporal restoration of ADAMTS13 activity and removal of pathogenic autoantibodies from the circulation. In case of persisting acquired TTP, adjunctive therapy with rituximab is used, a monoclonal antibody that eliminates all peripheral $\mathrm{B}$ cells by targeting their CD20 receptor. ${ }^{13}$ Rituximab improves treatment outcomes and has been shown to reduce relapse rates in acquired TTP. ${ }^{14-16}$ More recently, caplacizumab, a humanized anti-VWF nanobody that interferes with binding of platelets to VWF is currently being tested in clinical trials and has been shown to effectively contribute to fast resolution of acute TTP episodes. ${ }^{17}$ The majority of current therapies focus on the elimination of autoantibodies toward ADAMTS13, but they do not resolve the underlying autoimmune pathogenesis of acquired TTP. Likewise, our knowledge on triggers that induce relapses in patients with acquired TTP is very limited and prognostic markers for prediction of relapses have not been identified. Thus, more precise therapeutics and diagnostic methods must therefore be developed to further improve TTP management. ${ }^{18}$

The ADAMTS13 gene C9ORF8 is located on chromosome $9 \mathrm{q} 34 .^{19,20}$ The gene spans $37 \mathrm{~kb}$ and contains 29 exons. The ADAMTS13 protein consists of 1,427 amino acid residues, which includes a signal peptide and a propeptide. Hepatic stellate cells produce the majority of the circulating ADAMTS13. ${ }^{21}$ The posttranslationally modified and glycosylated ADAMTS13 that can be purified from human plasma has a molecular weight of 190 $\mathrm{kDa}^{20,22,23}$ and lacks the signal peptide and propeptide. ${ }^{24}$

The available three-dimensional structures for ADAMTS13 (Protein Data Bank ID: 3GHM, 3GHN, 3VN4) only partially span the full protein sequence, as the structure of many domains has not yet been resolved through experimental methods. The unavailable structures include those of the metalloprotease (Mp), thrombospondin type-1 repeats (TSR2-8) and CUB domains $^{22,25}$ as shown in -Fig. 1 (A, B). In this paper, we complement the available experimental structures with modeled structures of the metalloprotease domain, which contains the active site of ADAMTS13, and of the CUB1 and CUB2 domain, two C-terminal domains which are known to play a major role in the regulation of the expression of ADAMTS13 enzymatic activity.

The design of novel treatment and diagnostic strategies requires a detailed understanding of the structure-function relationships of ADAMTS13. This paper highlights our current knowledge on the ADAMTS13 protein structure and its structure-function relationships and provides insights into the structure of thus far unexplored ADAMTS13 domains based on homology modelling.

A detailed description of the structure-function relationships of ADAMTS13 as presented in this review provides a basis for the development of rationally designed therapeutics for treatment of patients with acquired TTP that specifically take into account the structural features of ADAMTS13 and the mode by which its activity is regulated in vivo.

\section{Propeptide}

ADAMTS13 and all other ADAMTS proteases, belonging to the same ADAMTS protein family, contain a propeptide. ${ }^{26}$ The propeptide of many metalloproteases contains a cysteineresidue that binds to the zinc ion present in the catalytic cleft of the metalloprotease domain. This interaction has been suggested to block the active site, thereby maintaining the protease in its zymogen form. ${ }^{27}$ Following the cleavage of the propeptide, the interaction of the cysteine with the zinc ion is lost resulting in conversion of the zymogen to its active form, in which the active site of metalloprotease becomes accessible for its substrate. Activation via this so-called cysteine switch occurs following cleavage of the propeptide at its terminal Arg-X-Arg/Lys-Arg motif by furin proteases. ${ }^{28}$ Cysteine switchmediated activation is considered to occur in every ADAMTS family member, as well as in members of ADAMs, the matrix metalloproteases (MMPs) and snake venom metalloproteases 
A

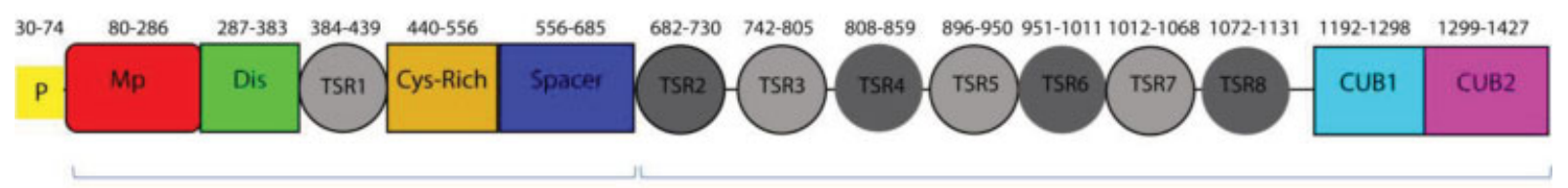
$\begin{array}{ll}\text { Proximal domains (MDTCS) Distal domains } & \text { D }\end{array}$

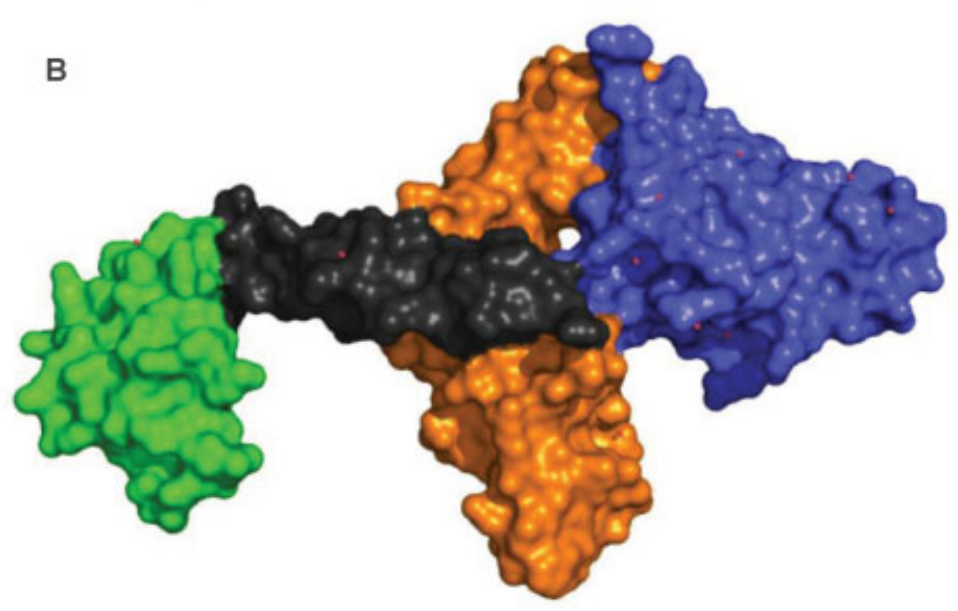

Fig. 1 (A) Domain organization of ADAMTS13. (B) Crystal structure of the DTCS domains of ADAMTS13 (Protein Data Bank ID: 3GHM); green surface represents the Dis domain; black structure is the TSR1 domain; yellow structure is Cys-rich domain and blue structure is the spacer domain. Of note, TTP antibodies have been described to target each of these protein domains, while those that bind to the spacer region correspond to the majority of these $(90 \%) .{ }^{60}$

(SVMPs). ${ }^{29}$ Interestingly, the conserved "cysteine-switch" found in other metalloproteases is not present in the propeptide of ADAMTS13, suggesting that a cysteine-switch does not control the catalytic activity of ADAMTS13. In agreement with this, uncleaved pro-ADAMTS13 secreted from furin-deficient cells is also capable of processing VWF. ${ }^{24}$ The ADAMTS13 propeptide is relatively short (45 amino acids) and displays little homology to the propeptide of other ADAMTS proteases. Therefore, the propeptide is most likely not involved in the activation of the catalytic activity of ADAMTS13.

\section{Metalloprotease Domain}

The metalloprotease (Mp) domain of ADAMTS13 contains several features that are crucial for its ability to cleave its substrate VWF. To study these features, we constructed a homology model of the Mp domain with the disintegrin-like domain (-Fig. 2A). The metalloprotease (Mp) domain contains three histidine residues that are responsible for the coordination of a $\mathrm{Zn}^{2+}$ ion inside the catalytic cleft $^{30}$ ( - Fig. 2A, B). $\mathrm{Zn}^{2+}$ interacts with the active site residue, Glu225, to facilitate substrate cleavage. His234Gln mutation in $\mathrm{Zn}^{2+}$ binding motif results in congenital TTP due to loss of coordination on the $\mathrm{Zn}^{2+}$ ion. ${ }^{31}$

Another signature-feature of the Mp domain is the highly conserved Met249 residue (- Fig. 2B). The Met249 turns the backbone of the Mp domain near the active site into the socalled Met-turn towards the C-terminus of the HEXXHXXGXXH sequence (residues 224-234) that coordinates binding of the $\mathrm{Zn}^{2+}$ ion. ${ }^{30}$ This Met-turn provides a relatively hydrophobic environment for the three active site histidine residues and the corresponding bound $\mathrm{Zn}^{2+}$ ion as illustrated in - Fig. 2B. The highly conserved Met-turn and the active site zinc binding motif gave birth to the term "metzincins," to denote the class of enzymes that contain the Met-turn $/ \mathrm{Zn}^{2+}$ motif. ${ }^{30}$ Replacement of Met249 by other residues is likely to impair molecular stability and activity. ${ }^{32}$ The stability of $\mathrm{Zn}^{2+}$ binding in Mp domain can be affected by mutations. The relatively conservative Ala250Val mutation in congenital TTP induces reduced ADAMTS13 secretion and reduced proteolytic activity. ${ }^{33}$

Previous studies that employed homology models ${ }^{34,35}$ as well as analysis of our carefully constructed structural model of the human ADAMTS13 Mp domain, presented in this paper, suggest the presence of three putative calcium binding sites. Site 1 and site 2 together form a calcium cluster which consists of connector loop, residues Glu83, Asp166, Asp168, Cys281 and Asp284 as shown in -Fig. 2C. These residues are also well conserved in ADAMTS1, ADAMTS4 and ADAMTS5. The calcium in site 3 is oriented by the side chains of Asp182 and Glu212 (- Fig. 2D) which are also conserved in the previously mentioned ADAMTS proteases. ${ }^{34,35}$ Furthermore, Leu183, Arg190 and Val192 contribute to the stabilization of the calcium ion ( $\mathbf{- F i g . ~ 2 C )} .{ }^{34,35}$ Mutation of site 3 residues Glu184, Asp187 and Glu212 to alanine resulted in a significant reduction of ADAMTS13 activity toward its substrate $\mathrm{VWF}^{34}$ Conceivably, an underlying reason for the drop in the activity is the vicinity of the site 3 loop to the active site of the Mp domain. This loop is closed by a disulphide bond in ADAMTS1, ADAMTS4 and ADAMTS5. ADAMTS13 lacks this disulphide bridge, but a salt bridge between Glu184 and $\operatorname{Arg} 190^{35}$ mediates closure of this calcium binding loop (-Fig. 2D). In one case of congenital TTP, Asp173 is mutated 

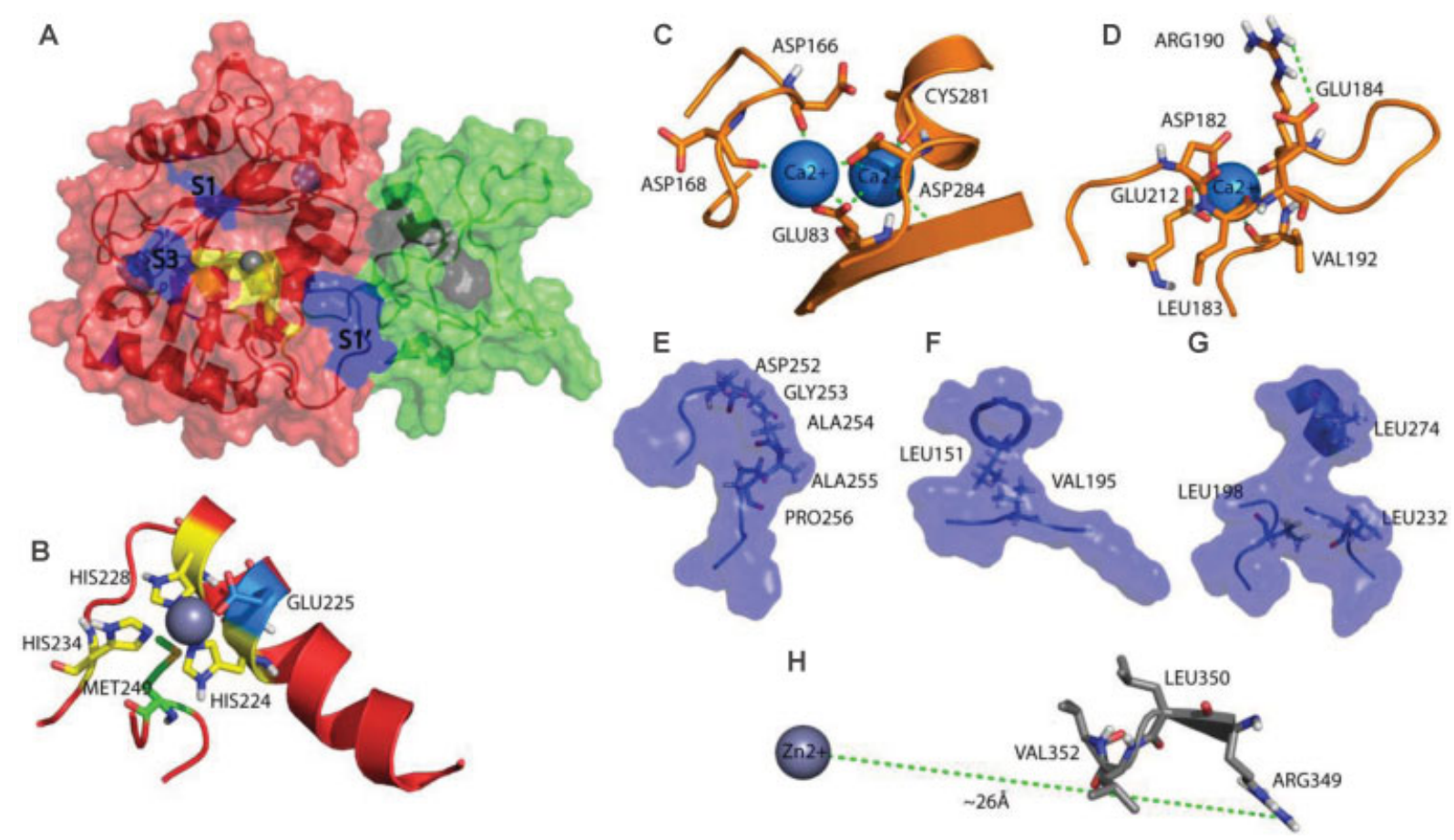

Fig. 2 (A) Homology model of metalloprotease domain (red) of ADAMTS13 together with disintegrin-like domain (green). Active site of metalloprotease is highlighted in yellow. Subsites are highlighted in blue and exosite on the disintegrin-like domain is highlighted in dark grey. (B) Residues coordinating Zn2+ at the active site of Mp domain; histidine residues are in yellow and the coordinated zinc is represented in grey sphere. The residue Met 249 that is causing the met-turn is coloured in light green and the active site residue Glu225 is highlighted in blue. $\mathrm{Zn}^{2+}$ interacts with residue Glu225 to polarize a water molecule ${ }^{30}$ needed to hydrolyze the scissile bond at Tyr1605-Met1606 in the A2 domain of VWF. (C) Calcium-binding sites 1 and 2; blue spheres represent calcium ions and residues that are highlighted in orange sticks are the calcium-binding residues of metalloprotease domain. Sites 1 and 2 form calcium cluster. (D) Calcium-binding site 3, high affinity calcium binding site. (E) S1' subsite of Mp. (F) S1 subsite of Mp. (G) S3 subsite of Mp domain. (H) The distance between $\mathrm{Zn2}+$ at the Mp domain and exosite 1 in disintegrin-like domain; Arg349 and Leu350 residues of disintegrin-like domain are functioning as exosite 1 and $\operatorname{Arg} 349$ is around $26 \AA$ away from active site zinc ion.

to glycine. Asp173 is located in proximity of the calcium cluster at sites 1 and 2 and mutation of this site reduces the interface area between metalloprotease and disintegrin-like domain, causing improper folding and low ADAMTS13 secretion efficiency. ${ }^{35}$ In patients diagnosed with acquired TTP, the region Glu98-Leu108 was found to represent the epitope of causative autoantibodies. ${ }^{36}$ Inspection of our Mp domain homology model reveals that this epitope is located at the Nterminus of helix $\alpha 2$ which is also in the interface area between metalloprotease and disintegrin-like domain. Antibody binding to this area may result in overall conformational changes and/or changes in substrate binding which together may lead to a loss of activity.

The Mp domain variable regions (VRs) of ADAMTS family members are known to confer the substrate specificity for each

Table 1 Definition of the variable regions of the human ADAMTS13 metalloprotease domain and the effects of VR mutations on the proteolysis of human $\mathrm{VWF}^{37}$

\begin{tabular}{|l|l|l|}
\hline Variable regions & Residues & $\begin{array}{l}\text { Effects of mutations } \\
\text { in VWF proteolysis }\end{array}$ \\
\hline VR1 & Glu184-Arg193 & 2 -10-fold reduction \\
\hline VR2 & Phe216-Val220 & Minor importance \\
\hline VR3 & Gly236-Ala261 & No changes \\
\hline
\end{tabular}

family member. There are three VRs in the Mp domain of human ADAMTS13 which are summarized in - Table 1. The substrate VWF is recognized by the three subsites $\left(\mathrm{S} 1^{\prime}, \mathrm{S} 1, \mathrm{~S} 3\right.$ as shown in -Fig. 2E-G, respectively) of which residues are located at the vicinity of the VRs. ${ }^{37}$ Mutations of Thr196Ile ${ }^{38}$ near S1 site and Leu232 $\mathrm{Gln}^{39}$ in S3 site result in congenital TTP due to likely disruption of subsite interactions with VWF.

\section{Disintegrin Domain}

C-terminal of the Mp domain of ADAMTS13, a disintegrin-like (Dis) domain, is present. The modeled structure of the complex between the Mp and Dis domains of ADAMTS13 is shown in -Fig. 2A. Disintegrins are short soluble polypeptides which consist of 47 to 84 amino acid residues and belong to a family of platelet aggregation inhibitors, capable of binding to a platelet surface protein called GPIIb/IIla, which are widely found in snake venom proteins. ${ }^{40} \mathrm{~A}$ common feature shared by disintegrins is the presence of a RGD sequence that is found on a conserved hairpin loop which allows for high-affinity binding to integrin receptors such as GPIIb/IIIa. ${ }^{41}$

The ADAMTS13 Dis domain does not share structural homology with other disintegrin domains that are known to be interacting with integrins. The RGD tripeptide is not present in ADAMTS13 Dis domain, but sequences that are highly conserved with other members of ADAMTS family are 
present. This may indicate that through evolution from a common ancestor protein, the ADAMTS13 Dis domain has evolved along with its three-dimensional structure to function differently as compared with other disintegrins. The Dis domain of ADAMTS13 plays a role in combination with cysteine-rich and spacer domains of ADAMTS13 in the enhancement of substrate binding, ${ }^{42-44}$ as was previously shown for the metalloprotease-disintegrin domains. ${ }^{45}$

Previously published crystal structures of ADAMTS1, ADAMTS4 and ADAMTS5 show that the Mp domain is found in complex with the Dis domain. ${ }^{46,47}$ The Mp domain has a higher proteolytic activity when it is in complex with the Dis domain. ${ }^{48}$ During the functional characterization of exosites on the surface of ADAMTS13, a single point mutation R349D in the disintegrin-like domain, resulted in an almost complete loss of proteolytic activity. ${ }^{48}$ Further investigations have revealed that recombinant ADAMTS13 harbouring R349A and L350G mutations showed a 10 - to 20 -fold reduction in proteolytic activity, whereas V352G resulted in 4- to 5-fold reduction. Congenital TTP patients have been described to carry a Arg349Cys ${ }^{49}$ mutation in exosite- 1 and Pro353 Leu $^{50}$ near exosite-1. These mutations cause impaired ADAMTS13 secretion by 24 and $69 \%$, respectively, and result in a significant decrease by 99.5 and $97 \%$ in VWF proteolysis, respectively, due to diminished interactions between exosite-1 and VWF. An epitope on the Dis domain is available between 332 and 364 that spans the exosite- 1 residues. ${ }^{51}$ Binding of autoantibodies to this epitope may directly prevent the interaction of exosite1 residues with VWF. In the light of these findings, Arg349 was hypothesized to interact with Asp1614 in the VWFA2 domain. This hypothesis relies on an approximate $26 \AA$ distance both between Arg349 and the $\mathrm{Zn}^{2+}$ ion in the catalytic cleft and between Asp1614 and the cleavage site in VWF which is between Tyr1605 and Met1606. ${ }^{43}$ The distance of approximately $26 \AA ̊$ between $\mathrm{Arg} 349$ and $\mathrm{Zn}^{2+}$ ion is also observed in our model (-Fig. 2H).

\section{Cysteine-Rich Domain}

Cysteine-rich domains (CRDs) are present in a variety of different proteins, where this domain may perform different functions. In ADAMTS13, a single CRD, the crystal structure of which is displayed in - Fig. 3A, is located between the first thrombospondin type- 1 repeat and spacer domain (-Fig. 1A). The ADAMTS13 CRD contains 10 cysteine residues which are all paired to form disulphide bonds. ${ }^{22}$

The ADAMTS13 CRD shows homology to CRDs present in other ADAMTS proteases. However, a part of the sequence of the ADAMTS13 CRD is not conserved as compared with other ADAMTS family members and was found to represent a functional part of the domain. This non-conserved region in the CRD lies between the residues Ala451 and Ser501 and harbours the exosite-2 amino acid residues: Gly471-Ala472-Ala473-Val474, just preceding the so-called V-loop (residues 474-481) as shown in - Fig. 3A, B. These residues form a pocket that favours hydrophobic interactions with Ile1642, Trp1644, Ile1649, Leu1650 and Ile1651 in the VWF-A2 domain which become exposed upon shear stress induced unfolding (-Fig. 3D) ${ }^{44}$ The

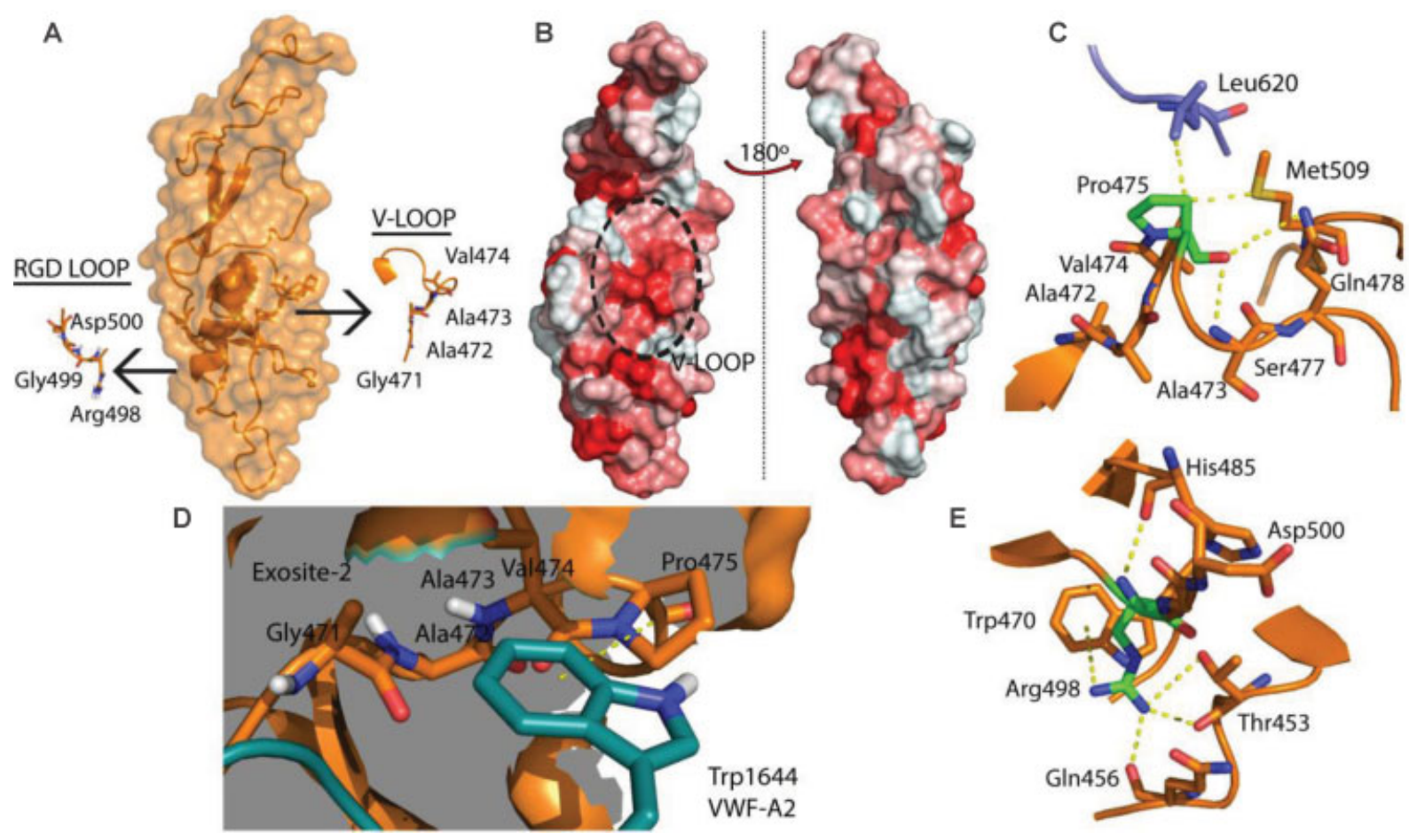

Fig. 3 (A) CRD of ADAMTS13 with V- and RGD-loops. (B) Surface representation of CRD of coloured according to hydrophobic properties: dark red $=$ more hydrophobic. Shown are two orientations of the CRD after a $180^{\circ}$ rotation about the vertical axis. Exosite- 2 residues which are forming a hydrophobic pocket are labelled. (C) Intradomain interactions of Pro475 residue from the CRD (green sticks) with residues from the CRD domain (orange sticks) and Leu620 from spacer domain (blue sticks) in ADAMTS13. (D) A likely binding mode of VWF A2 domain to ADAMTS13 CRD where Pro475 seems to interact with Trp1644. (E) The RGD loop of ADAMTS13 CRD. 
CRD domain contains epitopes for autoantibodies, but the epitope sequences are not well characterized yet. ${ }^{51}$

The hydrophobic pocket (shown in - Fig. 3C) within the CRD is adjacent to Pro475. A single nucleotide polymorphism Pro475 is common in the Japanese population ${ }^{52}$ and is associated with reduced plasma ADAMTS13 activity (by $16 \%$ ) and a twofold reduction in substrate affinity. ${ }^{52}$ Structural comparison of WT and Pro475Ser variant (PDB ID: $3 \mathrm{GHM}$ and 3VN4, respectively) reveals a slight difference of conformation of the V-loop. ${ }^{22,25}$ An overall root-meansquare deviation (RMSD) of $0.6 \AA$ is observed when comparing the crystal structure of the V-loop of WT and Pro475Ser ADAMTS13 which may result in reduced functionality for this part of the protein. Moreover, Pro475 in the V-loop forms hydrogen bonds with Ser477 and Gln478 as displayed in -Fig. 3C and makes van der Waals interactions with surrounding residues such as Val474 at the hydrophobic pocket, Met509 from the CRD and Leu620 from the spacer domain, which are predicted to be lost upon mutation. Further investigations employing a structural perspective have suggested that proline residues especially when present as cisisomers favour aromatics-proline interactions, particularly with electron-rich aromatics. ${ }^{53}$ In line with this, the cisproline at position 475 is predicted to interact with aromatic side chain of Trp1644 residue of VWF which is known to be interacting with the recently discovered hydrophobic pocket of CRD. A likely binding mode between Pro475 of ADAMTS13 and Trp1644 residues of VWF is shown in - Fig. 3D.

As previously mentioned, the RGD sequence, which is presented in several disintegrin domains, but not in that of ADAMTS13, is located in the CRD. Mutagenesis studies revealed that the RGD sequence is not involved in VWF proteolysis. ${ }^{54}$ This is an expected outcome considering the recently discovered hydrophobic pocket which is located on the opposite site of the RGD loop. Structural analysis of the ADAMTS13 crystal structure reveals that the side chain of the Arg498 in the RGD loop is not surface exposed ${ }^{22}$ but instead forms hydrogen bonds with the surrounding Thr453, Gln456 and His485 residues and cation-pi interactions with Trp470, which shield the RGD sequence from interaction (-Fig. 3E).

\section{Spacer Domain}

The ADAMTS13 spacer domain, the crystal structure of which is shown in - Fig. 4A, is a 130-amino acid long stretch between the CRD and the second thrombospondin type 1 repeat (see also - Fig. 1A). The spacer region is known to mediate several crucial interactions required for full activity of ADAMTS13. MDTCS, an ADAMTS13 variant that is truncated after the spacer domain, shows proteolytic activity toward VWF, but complete deletion of the spacer domain, as in the so-called MDTC variant, has a significantly reduced VWF processing activity. ${ }^{42,55}$ The crystal structure of ADAMTS13 shows that exosite- 3 is located in the spacer domain which contains a cluster of hydrophobic residues. ${ }^{22}$ These hydrophobic residues are decorated by polar and charged residues which present their side chains to the solvent. These surface-exposed residues, located on $\beta$-loops of the spacer domain, have been shown to mediate binding of ADAMTS13 to the A2 domain of VWF. $^{56,57}$ This surface-exposed region has been identified as being a major epitope of anti-ADAMTS13 antibodies that develop in patients with acquired TTP. ${ }^{56,58-60}$

Alanine mutation of the surface-exposed amino acids Arg660, Tyr661 and Tyr665 impairs the recognition of ADAMTS13 by VWF. ${ }^{56}$ These residues are also recognized by anti-ADAMTS13 antibodies that develop in a majority of patients with immune TTP. More detailed binding studies revealed that Arg568 and Phe592 also contribute to the epitope of anti-spacer domain-directed antibodies. ${ }^{60}$ These residues form exosite- 3 in the spacer domain. Conservative substitution of exosite-3 residues Arg568Lys/Phe592Tyr/ Arg660Lys/Tyr661Phe/Tyr665Phe leads to a gain-of-function variant of ADAMTS13 (GoF-ADAMTS13) which expresses higher activity toward VWF. ${ }^{61}$ The altered hydrophobicity and differently positioned side chains result in a ADAMTS13 variant which is resistant to autoantibodies which target this specific epitope. When considering the GoF mutant protein as a potential TTP therapeutic, resistance to anti-ADAMTS13 autoantibodies is a desired effect, but an increase in proteolysis of VWF multimers by GoF-ADAMTS13 may result in bleeding due to a less efficient recruitment of platelets at sites

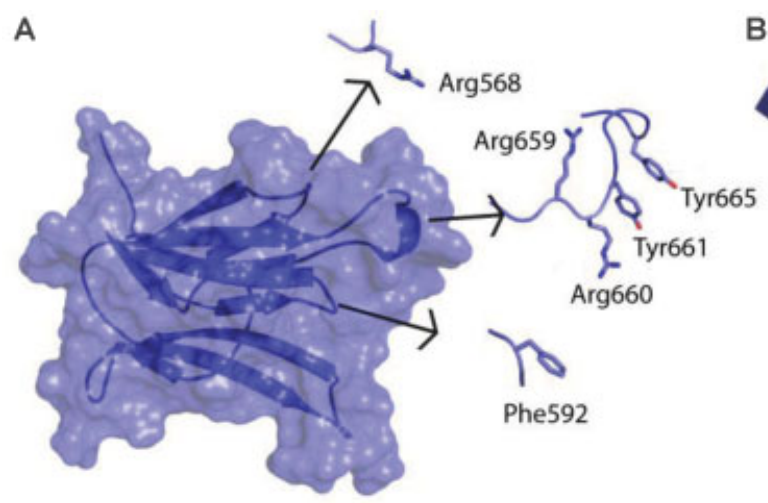

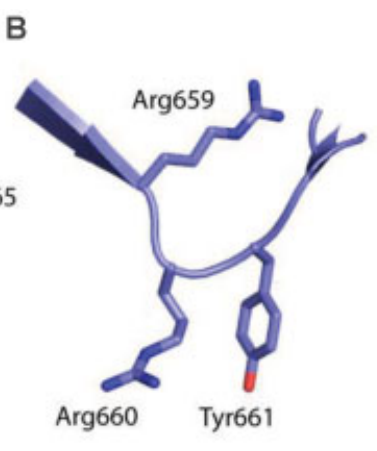

ADAMTS13-SPACER

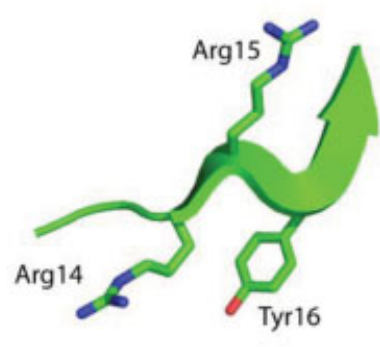

HNP1 - 3LO2

Fig. 4 Spacer domain of ADAMTS13. (A) Arrows indicating sites that are important both in VWF and autoantibody binding residues. (B) RRY sequences of ADAMTS13 spacer domain and HNP1 (PDB: 3LO2). 
of vascular injury by low-molecular-weight VWF multimers. Availability of a structural model for the full length ADAMTS13 protein, combined with a deepened insight into the regulatory mechanisms of ADAMTS13 activity, however, enables the rationalized design of other variants which combine a normal activity with reduced autoantibody recognition.

A recent study has shown that human neutrophil peptides (HNPs) and ADAMTS13 share a RRY motif that is crucial for binding of VWF to the ADAMTS13 spacer domain. ${ }^{62} \mathrm{~A}$ comparison of the RRY motif of the ADAMTS13 spacer domain and HNP1 is shown in - Fig. 4B. HNPs are abundantly released from activated neutrophils at sites of inflammation, and high concentrations of HNPs are detected in patients with TTP. ${ }^{63}$ In vitro study has shown that HNPs can compete with ADAMTS13 for binding to Glu1660-Asp1663 in VWF. ${ }^{62}$ These findings suggest that release of HNPs by activated neutrophils may promote TMA under conditions of reduced plasma levels of ADAMTS13 such as observed in patients with congenital or acquired TTP. ${ }^{63}$

The VWF interaction landscape of the ADAMTS13 MDTCS domains is summarized in - Fig. $\mathbf{5 A}$ and includes the subsites from the $\mathrm{Mp}$ domain and exosites from the ancillary domains, along with the recently identified N-glycosylation sites. ${ }^{23}$ Removal of $\mathrm{N}$-glycans (shown as the yellow surfaces in - Fig. 5A) on the proximal MDTCS domains affects the rate of VWF cleavage. $\mathrm{N}$-glycosylation sites in Mp domain are located in the vicinity of both the catalytic cleft and the site 3 $\mathrm{Ca}^{2+}$ ion, and the removal of these glycans reduces the rate of VWF proteolysis. ${ }^{64} \mathrm{~N}$-glycosylation by itself does not have a significant effect on protein structure, but it is known to contribute to protein expression and stability. ${ }^{65}$ The corresponding binding sites of ADAMTS13 MDTCS domains on the unfolded VWF A2 domain are summarized in - Fig. 5B.

\section{Thrombospondin Type-1 Repeats}

Thrombospondin type- 1 repeats (TSRs) have been identified in a large number of proteins involved in extracellular matrix organization. $^{66}$ TSRs are composed of two antiparallel strands (-Fig. 6A) and their crystal structure shows the presence of conserved tryptophan residues that are solvent-exposed to facilitate ligand interaction ${ }^{67}$ as shown in -Fig. 6B for TSR1 of ADAMTS13.

All members of the ADAMTS family contain TSR domains and yet the function of the TSRs in ADAMTS13 is still not well understood. The crystal structure of TSR1, which is located between the Dis domain and CRD, has been determined. ${ }^{22}$ There are eight TSR domains in ADAMTS13 and three linker

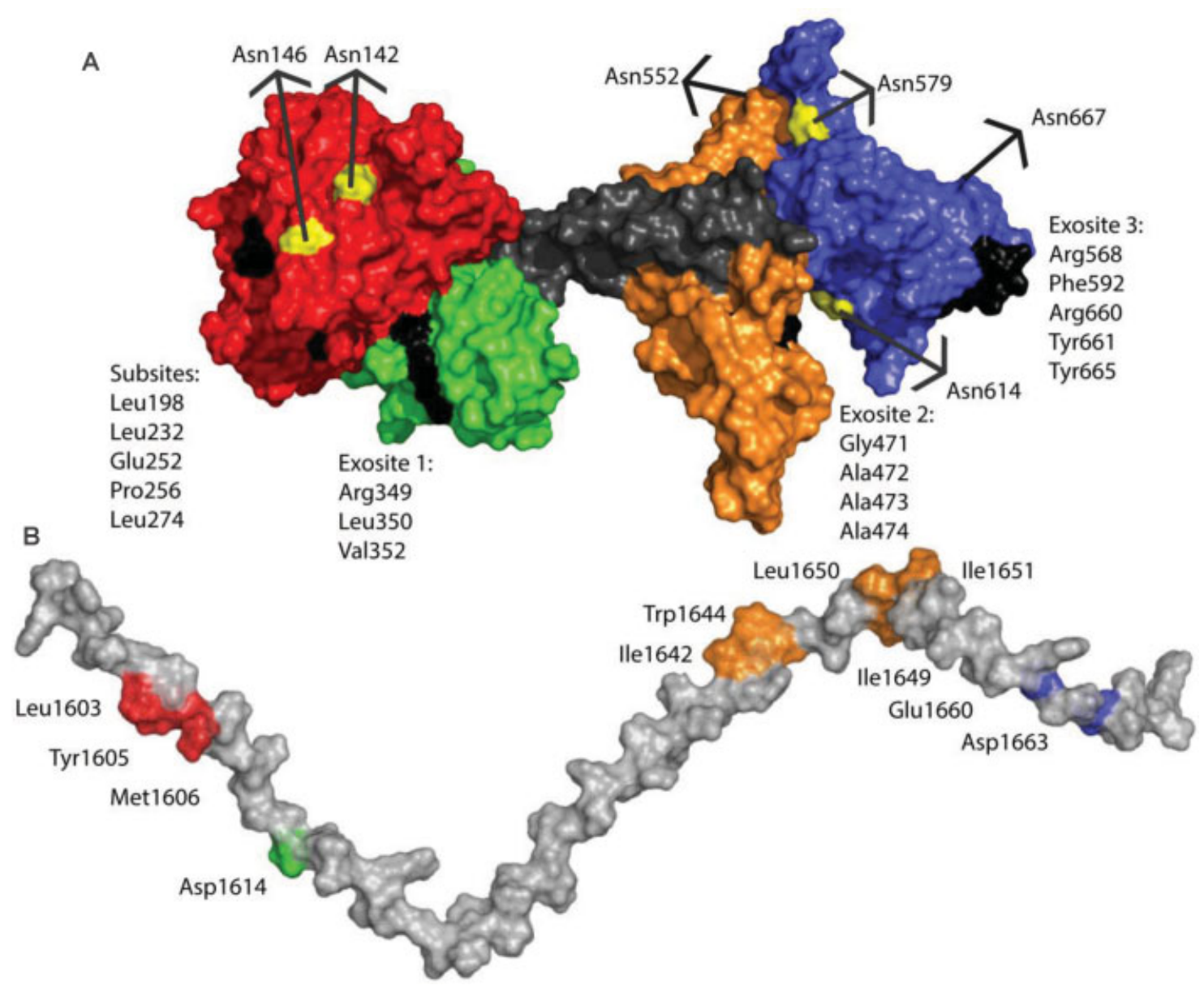

Fig. 5 ADAMTS13 and VWF interactions. (A) MDTCS model of ADAMTS13. Surface in red is Mp domain, green is Dis domain, dark grey is TSR1, orange is CRD, dark blue is spacer domain. Black surfaces on MDTCS model point out the location of the subsites and exosites. Yellow surfaces represent the Nglycosylation sites on the MDTCS model. Highlighted in red, Pro475 is a cis-isomer which is likely to interact with aromatic side chain of Trp1644. (B) Model of unravelled VWF A2 domain, showing ADAMTS13 binding sites with the corresponding surface colour for each domain in MDTCS model. 


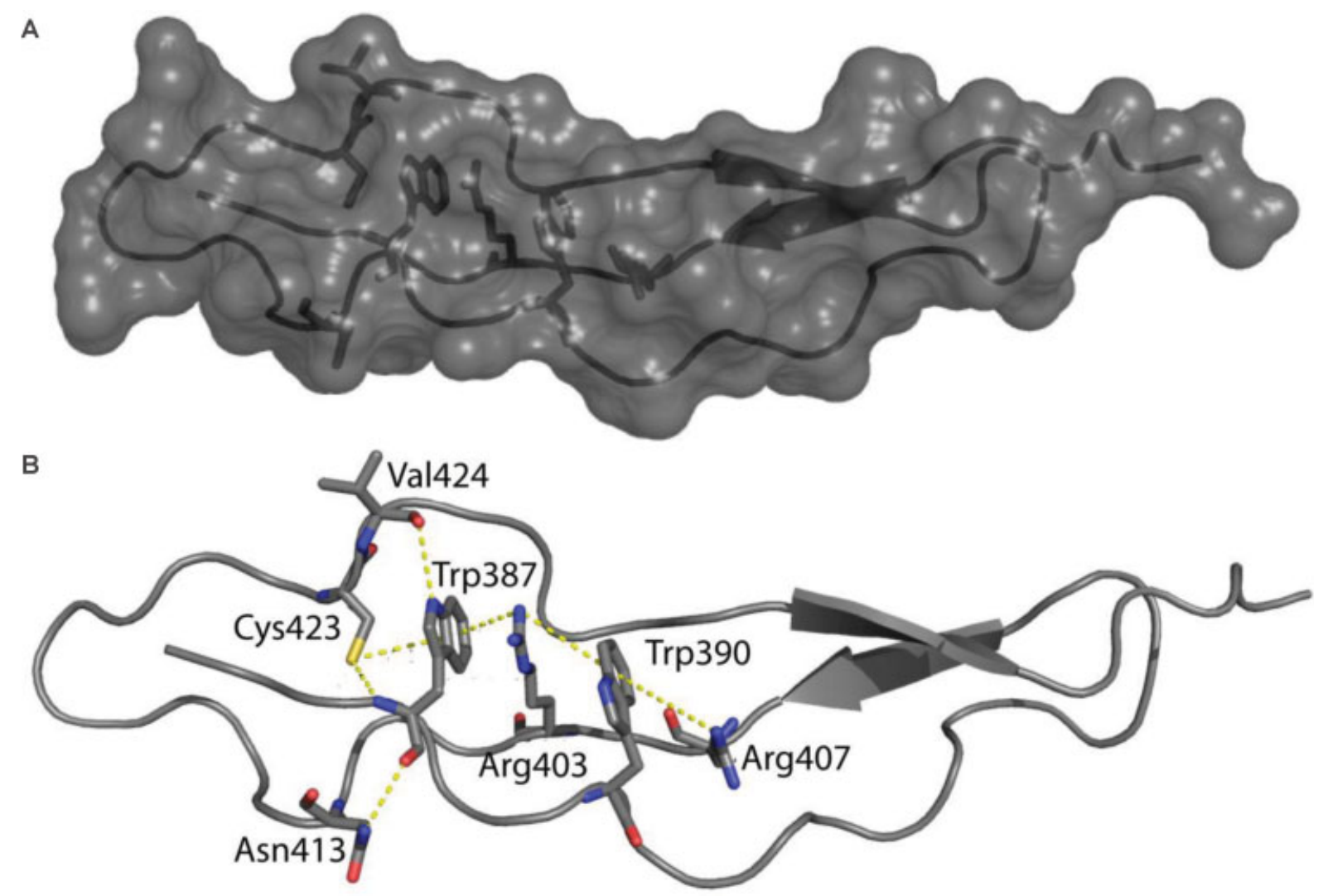

Fig. 6 (A) TSR1 domain of ADAMTS13. (B) WXXW motif on the TSR1 domain of ADAMTS13 and its intraprotein interactions. Trp387 forms hydrogen bonds with Asn413, Cys423, Val424, cation-pi interaction with Arg409 and aromatic-sulphur interactions with Cys423. Trp390 forms only cation-pi interactions with Arg407 and Arg409.

regions exist between TSR2/TSR3, TSR4/TSR5 and TSR8/CUB1 domains. ${ }^{20,68}$

O-fucosylation is a common post-translational modification of TSRs that has been found in ADAMTS13. ${ }^{23,69,70}$ In the absence of TSR O-fucosylation of ADAMTS13, cellular secretion is reduced by up to $100 \%$ most likely due to improper folding of the TSRs. ${ }^{23,69,70}$ Asn707 of TSR2 and Asn828 of TSR4 contain Nglycans. ${ }^{23}$ While substitution of these Asn residues for Gln has no effect on protein secretion, a decreased activity against the synthetic substrate FRETS-VWF73 under static conditions has been observed. When probed under flow conditions, these substitutions have no effect on substrate proteolysis. ${ }^{64}$ Nowak et al have explained this outcome by hypothesizing an increased flexibility at the linker regions in the absence of $\mathrm{N}$ glycosylation at these sites. ${ }^{64}$ Since the linker regions are important for ADAMTS13 flexibility, ${ }^{68}$ loss of N-glycosylation may favour the presence of ADAMTS13-closed conformations, which are considered as inactive states. ${ }^{71}$

A WXXW motif, potentially involved in ligand binding or protein activation, ${ }^{72}$ is present in TSR1 of ADAMTS13 (shown in - Fig. 6B). In ADAMTS13, this sequence is present as Trp387-X-X-Trp390 and mutation of the Trp387 residue significantly reduces ADAMTS13 secretion and reduces the binding affinity of ADAMTS13 to denaturated VWF while it lowers the proteolytic activity of ADAMTS13 against the small synthetic substrate FRETS-VWF73. ${ }^{72}$ Trp387 of ADAMTS13 is modified by C-mannosylation which promotes protein secretion. ${ }^{23,73}$ The aromatic side chain of Trp387 and Trp390 bind the cationic side chain of Arg409 via cation-pi interactions, which is a significant contribution to protein stability. ${ }^{22,74} \mathrm{~A}$ similar interaction is also present between Trp390 and Arg407 as illustrated in - Fig. 6B. ${ }^{22,74}$ Mutations of these residues may seriously impair the stability of the TSR1 domain, thus causing a conformational change between the $\mathrm{Mp} / \mathrm{Dis}$ and CRD/Spacer complexes. This hypothesis is confirmed by a congenital case of TTP, characterized by a Trp390Cys mutation, where the disruption of the WXXW motif with Trp390Cys mutation likely impairs both the stability of TSR1 domain and the C-mannosylation. One of the most commonly observed mutations in congenital TTP patients is Arg1060Trp in TSR7. This mutation does not affect protease activity but diminishes the protein secretion resulting in ADAMTS13 deficiency. ${ }^{75}$ All TSRs and the linkers contain epitopic regions where antibodies from both TTP patients and healthy donors target. ${ }^{36,51}$ However, none of these epitope sequences are shared or well characterized. Thus, the contribution of these epitope regions to the autoimmune course of TTP needs further study.

The three-dimensional structure of the MDTCS domains reveals the presence of multiple intramolecular disulphide bonds. ${ }^{22}$ Of note, Yeh et al and Bao et al proposed that free thiols are present in the distal TSR and CUB domains of ADAMTS13; these domains have been suggested to play an important role in the inhibition of platelet aggregation by 
reducing disulphide bonds of VWF multimers under shear stress. ${ }^{7,77}$ Despite the experimental results and suggested function for these free thiols, deposited structures of TSRs and CUB domains from other proteins which are available from the Protein Data Bank do not contain free cysteines.

\section{CUB Domains}

The acronym CUB stands for complement $\mathrm{C} 1 \mathrm{r} / \mathrm{C} 1 \mathrm{~s}$, Uegf (epidermal growth factor-related sea urchin protein) and Bmp1 (bone morphogenetic protein 1). ${ }^{78}$ Among all ADAMTS proteases, only ADAMTS13 contains CUB domains and these are located at the C-terminus of the enzyme ( - Fig. 1A). ADAMTS13 contains two homologous CUB domains (-Fig. 7A) which consist of 107 and 129 residues, respectively. An experimental structure is not available, but homology models of the ADAMTS13 CUB domains suggest that CUB1 and CUB2 domains are likely connected with a linker which starts from Leu1290 and ends at Glu1298 (shown in green in - Fig. 7A, connecting the cyan and purple CUB domains). Due to the flexibility of the linker region, the orientation of the two ADAMTS13 CUB domains remains elusive. The homology model of CUB2 domain suggests an unstructured region at the $\mathrm{C}$-terminus of ADAMTS13. This unstructured region (shown as yellow surface in - Fig. 7A) starts at Gln 1409 according to our model and ends at the last residue of ADAMTS13 Thr1427. A multiple sequence alignment (MSA) of ADAMTS13 CUB1 (data not shown) and CUB2 domains derived from different species shows a highly conserved sequence identity except in the unstructured $\mathrm{C}$ terminal region. A frameshift mutation (4143-4144insA) in ADAMTS13 CUB2 domain which causes congenital TTP due to loss of the last 49 amino acid of the protein results in impaired secretion. ${ }^{79}$ The apical sorting of ADAMTS13 is associated with this frameshift mutation $;{ }^{80}$ hence, the unstructured C-terminal end of the protein might direct apical release of ADAMTS13. A mutant ADAMTS13, Gly1239Val has been observed in congenital TTP patients and maintains a relative proteolytic activity of $66 \%$ as compared with WT-ADAMTS13. ${ }^{81}$ The Gly1239Val variant is, however, not secreted. Congenital TTP mutations in CUB domains impair the secretion of ADAMTS13, but do not completely abolish the proteolytic activity. CUB domains are targeted by autoantibodies. These antibodies are found both in TTP patients and in healthy donors ${ }^{36}$ and they share an epitope between Pro1292 and Tyr1296 which structurally corresponds to the linker region between CUB1 and CUB2 domains in our homology model. However, the link between antibodies targeting CUB domains and the pathophysiology of acquired TTP remains still elusive.

By structural consensus, CUB domains contain two $\beta$-sheets to form a $\beta$-sandwich structure and two disulphide bridges to stabilize the entire domain. ${ }^{82}$ However, ADAMTS13 CUB1 domain contains five cysteine residues and the CUB2 domain contains two cysteine residues. MSA of the ADAMTS13 CUB1 domain amino acid sequence from different species shows that the homologous residues Cys1192, Cys1213, Cys1236 and Cys1254 are well conserved, while Cys1275 is not (data not shown). Replacement of consensus cysteine residues of ADAMTS13 CUB1 domain by serine results in reduced secretion upon expression of the isolated human ADAMTS13 CUB1 domain. ${ }^{83}$ However, mutation at Cys 1275 has no effect on secretion or VWF proteolysis. ${ }^{83}$ The structural consensus between CUB domain-containing proteins predicts the presence of two disulphide bonds, but CUB1 domain of ADAMTS13 has been experimentally shown to contain free thiols at Cys1192, ${ }^{76}$ Cys1213 ${ }^{76,77}$ and Cys $1275 .{ }^{76,77,83}$ Even though the consensus cysteine residues of ADAMTS13 CUB1 domain are conserved in MSA, disulphide bonds in the structure appear not to be conserved. These free thiols of non-conserved disulphides in homologous proteins are generally linked to a variety of structural properties. ${ }^{84}$ In ADAMTS13, free thiols of carboxyl terminus TSRs and CUBs can reduce disulphide bonds in plasma VWF multimers ${ }^{76}$ and inhibit ULVWF string formation and with platelet aggregation. ${ }^{77}$

The roles of the CUB domains in ADAMTS13 are not fully understood due to discrepant results from different studies. It was shown that C-terminal truncated versions of ADAMTS13 (MDTCS), lacking TSR2-7 and the CUB1-2 domains, is able to cleave VWF. ${ }^{42}$ Later, the recombinant CUB-1 of ADAMTS13 was found to inhibit the cleavage of ULVWF. ${ }^{85}$ In vivo mouse experiments indicate that the
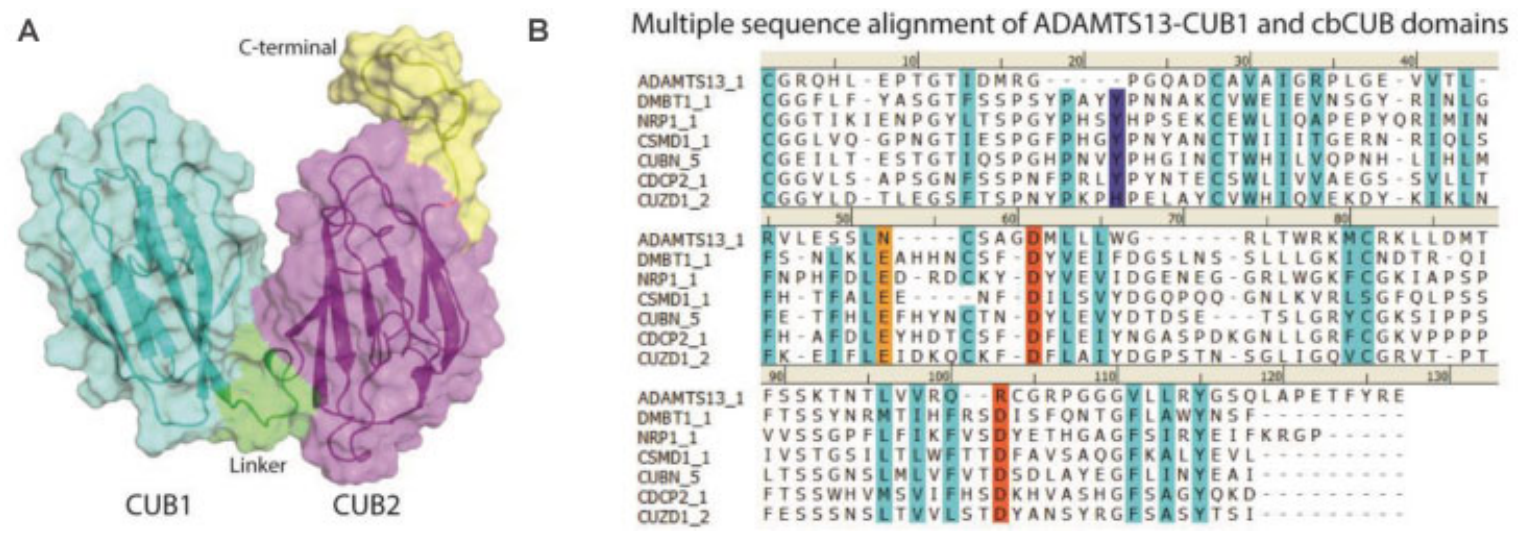

Fig. 7 ADAMTS13 CUB domains. (A) Homology model of ADAMTS13 CUB domains. Surface representation in cyan corresponds to CUB1, and in magenta corresponds to CUB2 domains. Surface in green points out the linker region which belongs to CUB1 domain, and surface in yellow shows the unstructured C-terminus of CUB2 domain. (B) Multiple sequence alignment of ADAMTS13-CUB1 domain with cbCUB domains. Light blue colour highlights the consensus sequence of CUB domains. The rest of the highlighted residues correspond to $\mathrm{Ca}^{2+}$ binding. 
ADAMTS13 CUB domains modulate in vivo thrombus formation. ${ }^{86}$ An ADAMTS13-binding site spans the D4-CK domains of VWF which interact with the C-terminal domains of ADAMTS13 following what has been described as a molecular zipper model binding mode. ${ }^{87-89}$

In CUB domains, calcium ions can be coordinated by clusters of negatively charged residues, to form calcium binding CUB (cbCUB) domains..$^{90}$ MSA of human ADAMTS13 CUB domains with cbCUB domains (shown in - Fig. 7B), however, shows incomplete conservation of calcium coordinating residues, whereby it is unlikely that the ADAMTS13 CUB1 domain will bind calcium.

\section{Open-Closed Conformations}

Allosteric regulation of ADAMTS13 by VWF was discovered after identification of autoantibodies in a TTP patient with increased ADAMTS13 activity. ${ }^{91}$ Electron microscopy (EM) and small angle X-ray scattering (SAXS) revealed that ADAMTS13 folds its distal CUB domains onto the CRD/spacer proximal domains to control exposure of exosites that are located in these domains (- Fig. 8A). ${ }^{91}$ When ADAMTS13 binds to the D4-CK domains of VWF, the protease becomes allosterically activated which subsequently promotes binding of ADAMTS13 to shear stress-induced unfolded VWF-A2 domain. ${ }^{89,91}$

When WT-ADAMTS13 is preincubated with anti-CUB monoclonal antibodies ${ }^{68}$ or VWF D4-CK domains, the activity of the protein significantly increases. ${ }^{91}$ In contrast, the activity of the earlier-mentioned GoF-ADAMTS13 (harbouring 5 amino acid substitutions in the spacer domain) is unaffected by anti-CUB autoantibodies, or VWF D4-CK. ${ }^{91}$ These observations indicate the involvement of residues Arg568, Phe592, Arg660, Tyr661 and Tyr665 in the binding of the spacer domain to the CUB domains in WT-ADAMTS13. EM defines the conformation of GoF-ADAMTS13 as elongated (open) and of WT-ADAMTS13, under low-shear condition, as condensed (closed). ${ }^{71}$ The closed conformation hides the major B-cell epitope in the spacer domain of ADAMTS13, such that the CUB domains protect ADAMTS13 from recognition by autoantibodies when it is in circulation. Only when ADAMTS13 adopts to its open conformation, it can form productive enzyme-ligand complexes, which are needed for expression of its proteolytic function. ${ }^{71}$

A recent study indicates that the closed conformation of ADAMTS13 controls the specificity of ADAMTS13 for its substrate VWF. ${ }^{92}$ GoF-ADAMTS13 is always in an elongated "open" conformation and was shown to cleave the A $\alpha$ chain of fibrinogen. ${ }^{92}$ Proteolysis of fibrinogen by GoF-ADAMTS13 affected fibrin formation and altered clot structure. ${ }^{92}$

The precise mechanism directing the conformational changes in activation of ADAMTS13 remains elusive. Previously mentioned linker regions which are located between distal TSRs and the CUB1 domain are responsible for flexibility ${ }^{68}$ The flexibility is no longer available in a mutant ADAMTS13 that lacks linker regions, such as to result in a less condensed conformation. ${ }^{68}$ Asn1235 and Asn1354 of ADAMTS13 CUB domains are $\mathrm{N}$-glycosylated (see - Fig. 7D). ${ }^{23}$ Substitution of these residues for glutamine increases the proteolytic efficacy of FRETS-VWF73 cleavage. ${ }^{64}$ It has been hypothesized that the absence of CUB domain glycans renders ADAMTS13 into its open, active, conformation. Nevertheless, how these glycans contribute to the conformational changes is unknown, yet removal of these glycans may alter the dynamics of the CUB domains which could change the distal loop flexibility or impair intraprotein interactions. ${ }^{77} \mathrm{~A}$ model for the conformational activation of ADAMTS13 has been proposed recently, where CUB1 and CUB2 domains are binding to the spacer domain but only CUB1 domain binding results in inhibition of proteolytic activity. ${ }^{93}$ The same model also shows the binding between VWF D4-CK and ADAMTS13 TSP8-CUB1-CUB2 domains. ${ }^{93}$

\section{Regulation of ADAMTS13 Activity}

Known tissue inhibitors of metalloproteinases (TIMP-1, -2, -3, -4) do not inhibit ADAMTS13 activity. ${ }^{94}$ In fact, natural inhibitors or activators/cofactors for ADAMTS13 have not been described. Instead, the conformational changes that are brought about by the intramolecular interactions between the CUB domains and the spacer region act like a self-inhibitory mechanism to control the activity of ADAMTS13. ADAMTS13 circulates in a state with low-activity and can only fully express its proteolytic activity towards VWF when the molecule changes from a closed to an open conformation, coinciding with exposition of substrate binding sites that are cryptic in the closed conformation. Final expression of ADAMTS13 activity, however, may also depend on factors other than those that are intrinsic to the ADAMTS13 molecule itself. In certain von Willebrand disease (VWD) patients, ${ }^{95}$ increased affinity to platelet glycoprotein Ib binding or mutations in the VWF A2 domain ${ }^{96}$ may expose the cryptic VWF scissile bond, independently from shear stress. Other conditions under which ADAMTS13 activity appears to be enhanced are those where increased shear forces occur. These include use of extracorporeal circuits, or of left ventricular assist devices (LVAD), as are used in patients with

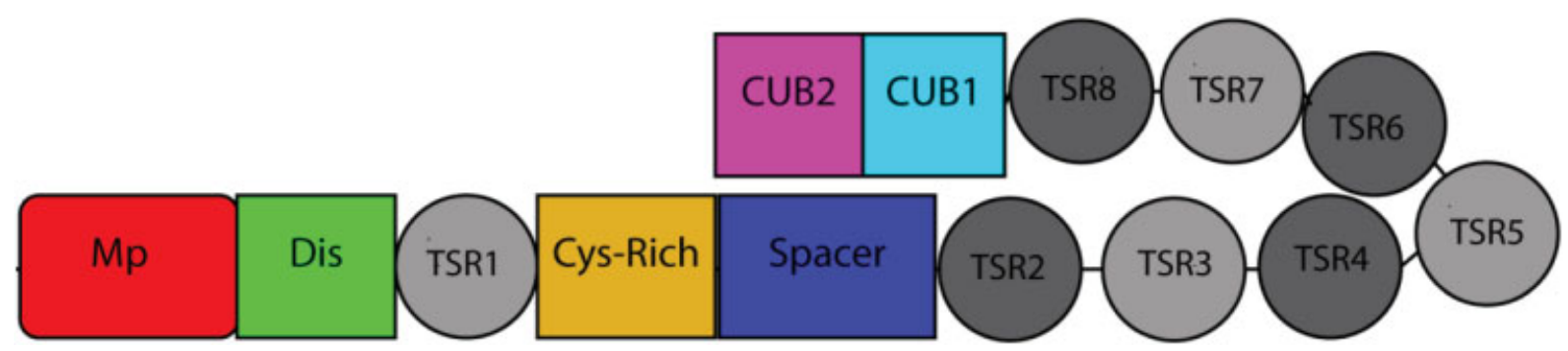

Fig. 8 A proposed domain organization of closed conformation of ADAMTS13. 
end-stage heart failure. ${ }^{97}$ Under such conditions, both spontaneous platelet binding and increased shear forces stimulate VWF A2 domain to unfold, which results in enhanced processing of VWF by ADAMTS13, which is independent of the ADAMTS13 structure.

Downregulation of ADAMTS13 activity may occur via proteolytic inactivation by plasmin, thrombin and activated factor $\mathrm{X}$, all of which are known to bind, cleave and inactivate ADAMTS13. ${ }^{98}$ Collectively these data suggest that ADAMTS13 binding of the CUB domains to the spacer domain controls the proteolytic activity of ADAMTS13 and proteolysis of ADAMTS13 itself may be involved in the physiological regulation of this metalloprotease. Details of both processes are not firmly established, however.

\section{ADAMTS13 and Thrombotic Thrombocytopenic Purpura}

Most of the autoantibodies in acquired TTP target the major epitope in the spacer domain in a manner which resembles the interactions between spacer and CUB domains in closed conformation. Autoantibody binding then prevents the binding of spacer exosite-3 to VWF A2 domain, which results in an inhibition of ULVWF processing. However, as also described in the earlier sections, inhibitory antibodies have been found to target virtually every domain in ADAMTS13. Binding of these autoantibodies to the proximal MDTCS domains is thought to interfere with the interaction between ADAMTS13 and VWF. Not only inhibitory antibodies are observed in TTP patients as some acquired TTP episodes present with noninhibitory antibodies that nonetheless can promote autoantibody-mediated ADAMTS13 depletion. ${ }^{99}$ In TTP patients, the presence of inhibitory or noninhibitory ADAMTS13 autoantibodies can cause ADAMTS13 deficiency, the major factor that determines the disease etiology in TTP. Further contributing factors to relapse of the disease likely involves a secondary trigger to reveal the phenotype of the TTP. ${ }^{100}$ Such trigger may constitute an infection or inflammation that follows the ADAMTS13 deficiency and which may initiate the disease onset. ${ }^{101}$ Such conditions also apply in congenital TTP, where most of the female individuals who are born with ADAMTS13 deficiency do not present the hallmarks of TTP until their first pregnancy. ${ }^{102}$ In these congenital TTP cases, a sudden increase in plasma VWF concentrations with the initiation of pregnancy were found to be a leading reason for the development of TTP. ${ }^{103}$ The prevalent lesson from the study of TTP patient cases and from experimental animal studies is that prevention of ADAMTS13 deficiency can be achieved through development of new treatment options that inhibit the occurrence of autoantibodies or prevent their binding to ADAMTS13. A complete understanding of the structure-function relationships of ADAMTS13 would facilitate the efficient development of such novel therapeutics. Structural bioinformatics and computational drug design methods are appropriate and instrumental to such development, even in the absence of experimental structures like X-ray crystallography or NMR spectroscopy. Simulation studies, employing accurate structural data, can further provide crucial information on intra- or intermolecular interactions of ADAMTS13 and can be used to study both congenital and acquired TTP disease mechanisms.

\section{Conclusions and Outlook}

In conclusion, the established three-dimensional structure of the DTCS domains and many experimental studies have provided insight into structure-function relationships of ADAMTS13. We complement this information by providing model structures to those domains for which no experimental structure is available. We present a comprehensive collection of structural data on ADAMTS13, including those of congenital and acquired TTP patients, and place these in a functional context. We observe that mutations or antibody binding to the proximal MDTCS domains may cause structural and functional changes in ADAMTS13, which result in a deficient processing of VWF. Regulation of ADAMTS13 activity is a complex and, yet, incompletely described process that involves allosteric regulatory mechanisms and conformational activation by VWF. Study of the open- and closed-conformation architectures of ADAMTS13 is expected to provide further insights into the functions of ADAMTS13, which are relevant to understanding the regulation of this important protease under both normal and pathological conditions, as in TTP.

The availability of detailed information on the structure and function of ADAMTS13, more in particular of the structural basis for the regulation of ADAMTS13 proteolytic activity, is of major importance for the rationalized structure-based development of therapies to treat TTP. With the availability of experimental and predicted structures for the full-length ADAMTS13 protein, experimentalists now are able to provide further comprehension on the regulation of VWF processing by ADAMTS13. Moreover, it creates the opportunity to exploit such structural information to provide new diagnostics and novel therapies for TTP patients. The development of a variety of different therapeutic approaches becomes feasible, including the engineering of antibody-bypassing ADAMTS13 variants, the design of molecules that are able to disrupt or compete with the interaction between autoantibodies and ADAMTS13, or the development of molecules (small molecules, peptides or antibodies) that can function as allosteric activators of ADAMTS13, even when the latter is in a closed conformation. Detailed knowledge about the three-dimensional structure of specific epitopes on the ADAMTS13 molecular surface, which is prone to the development of autoimmune responses or about the structure of frequently occurring antibodies themselves, is instrumental to such design.

\section{Materials and Methods}

Homology models of Mp and CUB domains of ADAMTS13 were built by use of the YASARA-Whatlf package. In short, for Mp domain of ADAMTS13, an inhibitor-bound crystal structure of ADAMTS4 (Protein Data Bank ID: 2RJP) ${ }^{46}$ and for CUB domains of ADAMTS13, a cubilin family CUB domain (Protein Data Bank ID: 3KQ4) were used as templates to generate homology models. The target-template sequences were aligned by 
YASARA and then manually inspected and adjusted for the correct structuring of the active site of the Mp domain. All models were refined by employing YASARA2 force field under 500ps molecular dynamics simulation. ${ }^{104}$ Model checks were performed with MolProbity. ${ }^{105}$ VWF A2 domain fragment was docked to ADAMTS13 CRD domain by HADDOCK. ${ }^{106}$

Coordinate files of the models that are presented in this paper are available from the authors upon request.

\section{Acknowledgements}

The research leading to these results has received funding from the Horizon 2020 Framework Program for Research and Innovation of the European Union under Grant Agreement No 675746.

\section{References}

1 Török TJ, Holman RC, Chorba TL. Increasing mortality from thrombotic thrombocytopenic purpura in the United Statesanalysis of national mortality data, 1968-1991. Am J Hematol 1995;50(02):84-90

2 Levy GG, Motto DG, Ginsburg D. ADAMTS13 turns 3. Blood 2005; 106(01):11-17

3 Moake JL. Thrombotic microangiopathies. N Engl J Med 2002;347 (08):589-600

4 Moschcowitz E. Hyaline thrombosis of the terminal arterioles and capillaries: a hitherto undescribed disease. Proc NY Pathol Soc 1924;24:21-24

5 Oka S, Nohgawa M. EB virus reactivation triggers thrombotic thrombocytopenic purpura in a healthy adult. Leuk Res Rep 2017;8:1-3

6 Mansouri Taleghani M, von Krogh AS, Fujimura Y, et al. Hereditary thrombotic thrombocytopenic purpura and the hereditary TTP registry. Hamostaseologie 2013;33(02):138-143

7 Oberic L, Buffet M, Schwarzinger M, et al; Reference Center for the Management of Thrombotic Microangiopathies. Cancer awareness in atypical thrombotic microangiopathies. Oncologist 2009;14(08):769-779

8 Matsuyama T, Uemura M, Ishikawa $M$, et al. Increased von Willebrand factor over decreased ADAMTS13 activity may contribute to the development of liver disturbance and multiorgan failure in patients with alcoholic hepatitis. Alcohol Clin Exp Res 2007;31(1, Suppl):S27-S35

9 Veyradier A, Obert B, Houllier A, Meyer D, Girma JP. Specific von Willebrand factor-cleaving protease in thrombotic microangiopathies: a study of 111 cases. Blood 2001;98(06):1765-1772

10 Coppo P, Adrie C, Azoulay E, et al. Infectious diseases as a trigger in thrombotic microangiopathies in intensive care unit (ICU) patients? Intensive Care Med 2003;29(04):564-569

11 Rieger M, Mannucci PM, Kremer Hovinga JA, et al. ADAMTS13 autoantibodies in patients with thrombotic microangiopathies and other immunomediated diseases. Blood 2005;106(04):1262-1267

12 Rock GA. Management of thrombotic thrombocytopenic purpura. Br J Haematol 2000;109(03):496-507

13 Boye J, Elter T, Engert A. An overview of the current clinical use of the anti-CD20 monoclonal antibody rituximab. Ann Oncol 2003; 14(04):520-535

14 Page EE, Kremer Hovinga JA, Terrell DR, Vesely SK, George JN. Rituximab reduces risk for relapse in patients with thrombotic thrombocytopenic purpura. Blood 2016;127(24):3092-3094

15 Westwood J-P, Webster H, McGuckin S, McDonald V, Machin SJ, Scully M. Rituximab for thrombotic thrombocytopenic purpura: benefit of early administration during acute episodes and use of prophylaxis to prevent relapse. J Thromb Haemost 2013;11(03): 481-490
16 Hie M, Gay J, Galicier L, et al; French Thrombotic Microangiopathies Reference Centre. Preemptive rituximab infusions after remission efficiently prevent relapses in acquired thrombotic thrombocytopenic purpura. Blood 2014;124(02): 204-210

17 Peyvandi F, Scully M, Kremer Hovinga JA, et al; TITAN Investigators. Caplacizumab for acquired thrombotic thrombocytopenic purpura. N Engl J Med 2016;374(06):511-522

18 George JN. How I treat patients with thrombotic thrombocytopenic purpura-hemolytic uremic syndrome. Blood 2000;96(04): 1223-1229

19 Gerritsen HE, Robles R, Lämmle B, Furlan M. Partial amino acid sequence of purified von Willebrand factor-cleaving protease. Blood 2001;98(06):1654-1661

20 Zheng X, Chung D, Takayama TK, Majerus EM, Sadler JE, Fujikawa K. Structure of von Willebrand factor-cleaving protease (ADAMTS13), a metalloprotease involved in thrombotic thrombocytopenic purpura. J Biol Chem 2001;276(44):41059-41063

21 Zhou W, Inada M, Lee TP, et al. ADAMTS13 is expressed in hepatic stellate cells. Lab Invest 2005;85(06):780-788

22 Akiyama M, Takeda S, Kokame K, Takagi J, Miyata T. Crystal structures of the noncatalytic domains of ADAMTS13 reveal multiple discontinuous exosites for von Willebrand factor. Proc Natl Acad Sci U S A 2009;106(46):19274-19279

23 Verbij FC, Stokhuijzen E, Kaijen PHP, van Alphen F, Meijer AB, Voorberg J. Identification of glycans on plasma-derived ADAMTS13. Blood 2016;128(21):e51-e58

24 Majerus EM, Zheng X, Tuley EA, Sadler JE. Cleavage of the ADAMTS13 propeptide is not required for protease activity. J Biol Chem 2003;278(47):46643-46648

25 Akiyama M, Nakayama D, Takeda S, Kokame K, Takagi J, Miyata T. Crystal structure and enzymatic activity of an ADAMTS-13 mutant with the East Asian-specific P475S polymorphism. J Thromb Haemost 2013;11(07):1399-1406

26 Brocker CN, Vasiliou V, Nebert DW. Evolutionary divergence and functions of the ADAM and ADAMTS gene families. Hum Genomics 2009;4(01):43-55

27 Van Wart HE, Birkedal-Hansen $\mathrm{H}$. The cysteine switch: a principle of regulation of metalloproteinase activity with potential applicability to the entire matrix metalloproteinase gene family. Proc Natl Acad Sci U S A 1990;87(14):5578-5582

28 Matthews DJ, Goodman LJ, Gorman CM, Wells JA. A survey of furin substrate specificity using substrate phage display. Protein Sci 1994;3(08):1197-1205

29 Stone AL, Kroeger M, Sang QXA. Structure-function analysis of the ADAM family of disintegrin-like and metalloproteinasecontaining proteins (review). (Review)J Protein Chem 1999;18 (04):447-465

30 Bode W, Gomis-Rüth FX, Stöckler W. Astacins, serralysins, snake venom and matrix metalloproteinases exhibit identical zincbinding environments (HEXXHXXGXXH and Met-turn) and topologies and should be grouped into a common family, the 'metzincins'. FEBS Lett 1993;331(1-2):134-140

31 Shibagaki Y, Matsumoto M, Kokame K, et al. Novel compound heterozygote mutations (H234Q/R1206X) of the ADAMTS13 gene in an adult patient with Upshaw-Schulman syndrome showing predominant episodes of repeated acute renal failure. Nephrol Dial Transplant 2006;21(05):1289-1292

32 Tallant C, García-Castellanos R, Baumann U, Gomis-Rüth FX. On the relevance of the Met-turn methionine in metzincins. J Biol Chem 2010;285(18):13951-13957

33 Uchida T, Wada $\mathrm{H}$, Mizutani $\mathrm{M}$, et al; Research Project on Genetics of Thrombosis. Identification of novel mutations in ADAMTS13 in an adult patient with congenital thrombotic thrombocytopenic purpura. Blood 2004;104(07):2081-2083

34 Gardner MD, Chion CK, de Groot R, Shah A, Crawley JT, Lane DA. A functional calcium-binding site in the metalloprotease domain of ADAMTS13. Blood 2009;113(05):1149-1157 
35 Lancellotti S, Peyvandi F, Pagliari MT, et al. The D173G mutation in ADAMTS-13 causes a severe form of congenital thrombotic thrombocytopenic purpura. A clinical, biochemical and in silico study. Thromb Haemost 2016;115(01):51-62

36 Grillberger R, Casina VC, Turecek PL, Zheng XL, Rottensteiner H, Scheiflinger F. Anti-ADAMTS13 IgG autoantibodies present in healthy individuals share linear epitopes with those in patients with thrombotic thrombocytopenic purpura. Haematologica 2014;99(04):e58-e60

37 de Groot R, Lane DA, Crawley JTB. The ADAMTS13 metalloprotease domain: roles of subsites in enzyme activity and specificity. Blood 2010;116(16):3064-3072

38 Levy GG, Nichols WC, Lian EC, et al. Mutations in a member of the ADAMTS gene family cause thrombotic thrombocytopenic purpura. Nature 2001;413(6855):488-494

39 Schneppenheim R, Budde U, Oyen F, et al. von Willebrand factor cleaving protease and ADAMTS13 mutations in childhood TTP. Blood 2003;101(05):1845-1850

40 Musial J, Niewiarowski S, Rucinski B, et al. Inhibition of platelet adhesion to surfaces of extracorporeal circuits by disintegrins. RGD-containing peptides from viper venoms. Circulation 1990; 82(01):261-273

41 Phillips DR, Charo IF, Parise LV, Fitzgerald LA. The platelet membrane glycoprotein IIb-IIIa complex. Blood 1988;71(04): 831-843

42 Zheng X, Nishio K, Majerus EM, Sadler JE. Cleavage of von Willebrand factor requires the spacer domain of the metalloprotease ADAMTS13. J Biol Chem 2003;278(32):30136-30141

43 de Groot R, Bardhan A, Ramroop N, Lane DA, Crawley JT. Essential role of the disintegrin-like domain in ADAMTS13 function. Blood 2009;113(22):5609-5616

44 de Groot R, Lane DA, Crawley JTB. The role of the ADAMTS13 cysteine-rich domain in VWF binding and proteolysis. Blood 2015;125(12):1968-1975

45 Blobel CP. Metalloprotease-disintegrins: links to cell adhesion and cleavage of TNF alpha and Notch. Cell 1997;90(04):589-592

46 Mosyak L, Georgiadis K, Shane T, et al. Crystal structures of the two major aggrecan degrading enzymes, ADAMTS4 and ADAMTS5. Protein Sci 2008;17(01):16-21

47 Gerhardt S, Hassall G, Hawtin P, et al. Crystal structures of human ADAMTS-1 reveal a conserved catalytic domain and a disintegrin-like domain with a fold homologous to cysteine-rich domains. J Mol Biol 2007;373(04):891-902

48 Gao W, Anderson PJ, Majerus EM, Tuley EA, Sadler JE. Exosite interactions contribute to tension-induced cleavage of von Willebrand factor by the antithrombotic ADAMTS13 metalloprotease. Proc Natl Acad Sci U S A 2006;103(50):19099-19104

49 Fujimura Y, Matsumoto M, Kokame K, et al. Pregnancy-induced thrombocytopenia and TTP, and the risk of fetal death, in Upshaw-Schulman syndrome: a series of 15 pregnancies in 9 genotyped patients. Br J Haematol 2009;144(05):742-754

50 Manea M, Kristoffersson A, Schneppenheim R, et al. Podocytes express ADAMTS13 in normal renal cortex and in patients with thrombotic thrombocytopenic purpura. Br J Haematol 2007;138 (05):651-662

51 Yamaguchi Y, Moriki T, Igari A, et al. Epitope analysis of autoantibodies to ADAMTS13 in patients with acquired thrombotic thrombocytopenic purpura. Thromb Res 2011;128(02): 169-173

52 Kokame K, Matsumoto M, Soejima K, et al. Mutations and common polymorphisms in ADAMTS13 gene responsible for von Willebrand factor-cleaving protease activity. Proc Natl Acad Sci U S A 2002;99(18):11902-11907

53 Zondlo NJ. Aromatic-proline interactions: electronically tunable $\mathrm{CH} / \pi$ interactions. Acc Chem Res 2013;46(04):1039-1049

54 Soejima K, Matsumoto M, Kokame K, et al. ADAMTS-13 cysteinerich/spacer domains are functionally essential for von Willebrand factor cleavage. Blood 2003;102(09):3232-3237
55 Tao Z, Wang Y, Choi H, et al. Cleavage of ultralarge multimers of von Willebrand factor by C-terminal-truncated mutants of ADAMTS-13 under flow. Blood 2005;106(01):141-143

56 Pos W, Crawley JTB, Fijnheer R, Voorberg J, Lane DA, Luken BM. An autoantibody epitope comprising residues R660, Y661, and Y665 in the ADAMTS13 spacer domain identifies a binding site for the A2 domain of VWF. Blood 2010;115(08):1640-1649

57 Jin SY, Skipwith CG, Zheng XL. Amino acid residues $\operatorname{Arg}(659)$, Arg (660), and $\operatorname{Tyr}(661)$ in the spacer domain of ADAMTS13 are critical for cleavage of von Willebrand factor. Blood 2010;115 (11):2300-2310

58 Luken BM, Turenhout EA, Hulstein JJ, Van Mourik JA, Fijnheer R, Voorberg J. The spacer domain of ADAMTS13 contains a major binding site for antibodies in patients with thrombotic thrombocytopenic purpura. Thromb Haemost 2005;93(02):267-274

59 Luken BM, Turenhout EA, Kaijen PH, et al. Amino acid regions 572-579 and 657-666 of the spacer domain of ADAMTS13 provide a common antigenic core required for binding of antibodies in patients with acquired TTP. Thromb Haemost 2006;96 (03):295-301

60 Pos W, Sorvillo N, Fijnheer R, et al. Residues Arg568 and Phe592 contribute to an antigenic surface for anti-ADAMTS13 antibodies in the spacer domain. Haematologica 2011;96(11):1670-1677

61 Jian C, Xiao J, Gong L, et al. Gain-of-function ADAMTS13 variants that are resistant to autoantibodies against ADAMTS13 in patients with acquired thrombotic thrombocytopenic purpura. Blood 2012;119(16):3836-3843

62 Pillai VG, Bao J, Zander CB, et al. Human neutrophil peptides inhibit cleavage of von Willebrand factor by ADAMTS13: a potential link of inflammation to TTP. Blood 2016;128(01): $110-119$

63 Cao W, Pham HP, Williams LA, et al. Human neutrophil peptides and complement factor $\mathrm{Bb}$ in pathogenesis of acquired thrombotic thrombocytopenic purpura. Haematologica 2016;101(11): 1319-1326

64 Nowak AA, O'Brien HER, Henne P, et al. ADAMTS-13 glycans and conformation-dependent activity. J Thromb Haemost 2017;15 (06):1155-1166

65 Lee HS, Qi Y, Im W. Effects of N-glycosylation on protein conformation and dynamics: Protein Data Bank analysis and molecular dynamics simulation study. Sci Rep 2015;5:8926

66 Adams JC, Tucker RP. The thrombospondin type 1 repeat (TSR) superfamily: diverse proteins with related roles in neuronal development. Dev Dyn 2000;218(02):280-299

67 Tan K, Duquette M, Liu JH, et al. Crystal structure of the TSP-1 type 1 repeats: a novel layered fold and its biological implication. J Cell Biol 2002;159(02):373-382

68 Deforche L, Roose E, Vandenbulcke A, et al. Linker regions and flexibility around the metalloprotease domain account for conformational activation of ADAMTS-13. J Thromb Haemost 2015; 13(11):2063-2075

69 Sorvillo N, Pos W, van den Berg LM, et al. The macrophage mannose receptor promotes uptake of ADAMTS13 by dendritic cells. Blood 2012;119(16):3828-3835

70 Ricketts LM, Dlugosz M, Luther KB, Haltiwanger RS, Majerus EM. O-fucosylation is required for ADAMTS13 secretion. J Biol Chem 2007;282(23):17014-17023

71 South K, Luken BM, Crawley JT, et al. Conformational activation of ADAMTS13. Proc Natl Acad Sci U S A 2014;111(52):18578-18583

72 Ling J, Su J, Ma Z, Ruan C. The WXXW motif in the TSR1 of ADAMTS13 is important for its secretion and proteolytic activity. Thromb Res 2013;131(06):529-534

73 Wang LW, Leonhard-Melief C, Haltiwanger RS, Apte SS. Posttranslational modification of thrombospondin type- 1 repeats in ADAMTS-like 1 /punctin-1 by C-mannosylation of tryptophan. J Biol Chem 2009;284(44):30004-30015

74 Gallivan JP, Dougherty DA. Cation-pi interactions in structural biology. Proc Natl Acad Sci U S A 1999;96(17):9459-9464 
75 Camilleri RS, Cohen H, Mackie IJ, et al. Prevalence of the ADAMTS-13 missense mutation R1060W in late onset adult thrombotic thrombocytopenic purpura. J Thromb Haemost 2008;6(02):331-338

76 Yeh HC, Zhou Z, Choi H, et al. Disulfide bond reduction of von Willebrand factor by ADAMTS-13. J Thromb Haemost 2010;8 (12):2778-2788

77 Bao J, Xiao J, Mao Y, Zheng XL. Carboxyl terminus of ADAMTS13 directly inhibits platelet aggregation and ultra large von Willebrand factor string formation under flow in a free-thiol-dependent manner. Arterioscler Thromb Vasc Biol 2014;34(02): 397-407

78 Bork P, Beckmann G. The CUB domain. A widespread module in developmentally regulated proteins. J Mol Biol 1993;231(02): 539-545

79 Pimanda JE, Maekawa A, Wind T, Paxton J, Chesterman CN, Hogg PJ. Congenital thrombotic thrombocytopenic purpura in association with a mutation in the second CUB domain of ADAMTS13. Blood 2004;103(02):627-629

80 Shang D, Zheng XW, Niiya M, Zheng XL. Apical sorting of ADAMTS13 in vascular endothelial cells and Madin-Darby canine kidney cells depends on the CUB domains and their association with lipid rafts. Blood 2006;108(07):2207-2215

81 Donadelli R, Banterla F, Galbusera M, et al; International Registry of Recurrent and Familial HUS/TTP. In-vitro and in-vivo consequences of mutations in the von Willebrand factor cleaving protease ADAMTS13 in thrombotic thrombocytopenic purpura. Thromb Haemost 2006;96(04):454-464

82 Romero A, Romão MJ, Varela PF, et al. The crystal structures of two spermadhesins reveal the CUB domain fold. Nat Struct Biol 1997;4(10):783-788

83 Zhou Z, Yeh HC, Jing H, et al. Cysteine residues in CUB-1 domain are critical for ADAMTS13 secretion and stability. Thromb Haemost 2011;105(01):21-30

84 Thangudu RR, Manoharan M, Srinivasan N, Cadet F, Sowdhamini $\mathrm{R}$, Offmann B. Analysis on conservation of disulphide bonds and their structural features in homologous protein domain families. BMC Struct Biol 2008;8:55

85 Tao Z, Peng Y, Nolasco L, et al. Recombinant CUB-1 domain polypeptide inhibits the cleavage of ULVWF strings by ADAMTS13 under flow conditions. Blood 2005;106(13):4139-4145

86 Xiao J, Jin SY, Xue J, Sorvillo N, Voorberg J, Zheng XL. Essential domains of a disintegrin and metalloprotease with thrombospondin type 1 repeats-13 metalloprotease required for modulation of arterial thrombosis. Arterioscler Thromb Vasc Biol 2011; 31(10):2261-2269

87 Feys HB, Anderson PJ, Vanhoorelbeke K, Majerus EM, Sadler JE. Multi-step binding of ADAMTS-13 to von Willebrand factor. J Thromb Haemost 2009;7(12):2088-2095

88 Crawley JTB, de Groot R, Xiang Y, Luken BM, Lane DA. Unraveling the scissile bond: how ADAMTS13 recognizes and cleaves von Willebrand factor. Blood 2011;118(12):3212-3221

89 Zanardelli S, Chion ACK, Groot E, et al. A novel binding site for ADAMTS13 constitutively exposed on the surface of globular VWF. Blood 2009;114(13):2819-2828
90 Andersen CBF, Moestrup SK. How calcium makes endocytic receptors attractive. Trends Biochem Sci 2014;39(02):82-90

91 Muia J, Zhu J, Gupta G, et al. Allosteric activation of ADAMTS13 by von Willebrand factor. Proc Natl Acad Sci U S A 2014;111(52): 18584-18589

92 South K, Freitas MO, Lane DA. Conformational quiescence of ADAMTS-13 prevents proteolytic promiscuity. J Thromb Haemost 2016;14(10):2011-2022

93 South K, Freitas MO, Lane DA. A model for the conformational activation of the structurally quiescent metalloprotease ADAMTS13 by von Willebrand factor. J Biol Chem 2017;292(14):5760-5769

94 Guo C, Tsigkou A, Lee MH. ADAMTS13 and 15 are not regulated by the full length and N-terminal domain forms of TIMP-1, $-2,-3$ and -4. Biomed Rep 2016;4(01):73-78

95 Holmberg L, Dent JA, Schneppenheim R, Budde U, Ware J, Ruggeri ZM. von Willebrand factor mutation enhancing interaction with platelets in patients with normal multimeric structure. J Clin Invest 1993;91(05):2169-2177

96 Hassenpflug WA, Budde U, Obser T, et al. Impact of mutations in the von Willebrand factor A2 domain on ADAMTS13-dependent proteolysis. Blood 2006;107(06):2339-2345

97 Jilma-Stohlawetz P, Quehenberger P, Schima H, et al. Acquired von Willebrand factor deficiency caused by LVAD is ADAMTS-13 and platelet dependent. Thromb Res 2016;137:196-201

98 Crawley JT, Lam JK, Rance JB, Mollica LR, O’Donnell JS, Lane DA. Proteolytic inactivation of ADAMTS13 by thrombin and plasmin. Blood 2005;105(03):1085-1093

99 Thomas MR, de Groot R, Scully MA, Crawley JT. Pathogenicity of anti-ADAMTS13 autoantibodies in acquired thrombotic thrombocytopenic purpura. EBioMedicine 2015;2(08):942-952

100 Vanhoorelbeke K, De Meyer SF. Animal models for thrombotic thrombocytopenic purpura. J Thromb Haemost 2013;11(Suppl 1): $2-10$

101 Fuchs TA, Kremer Hovinga JA, Schatzberg D, Wagner DD, Lämmle B. Circulating DNA and myeloperoxidase indicate disease activity in patients with thrombotic microangiopathies. Blood 2012; 120(06):1157-1164

102 Moatti-Cohen M, Garrec C, Wolf M, et al; French Reference Center for Thrombotic Microangiopathies. Unexpected frequency of Upshaw-Schulman syndrome in pregnancy-onset thrombotic thrombocytopenic purpura. Blood 2012;119(24): 5888-5897

103 Castaman G. Changes of von Willebrand factor during pregnancy in women with and without von Willebrand disease. Mediterr J Hematol Infect Dis 2013;5(01):e2013052

104 Krieger E, Joo K, Lee J, et al. Improving physical realism, stereochemistry, and side-chain accuracy in homology modeling: Four approaches that performed well in CASP8. Proteins 2009;77 (Suppl 9):114-122

105 Chen VB, Arendall WB III, Headd JJ, et al. MolProbity: all-atom structure validation for macromolecular crystallography. Acta Crystallogr D Biol Crystallogr 2010;66(Pt 1):12-21

106 van Zundert GCP, Rodrigues JPGLM, Trellet M, et al. The HADDOCK2.2 Web server: user-friendly integrative modeling of biomolecular complexes. J Mol Biol 2016;428(04):720-725 